\title{
The Coexistence and Decoupling of Bulk and Edge States in Disordered Two-dimensional Topological Insulators
}

\author{
Yan-Yang Zhang, ${ }^{1,2}$ Man Shen, ${ }^{1,3}$ Xing-Tao An, ${ }^{4}$ Qing-Feng Sun, ${ }^{5}$ Xin-Cheng Xie, ${ }^{5}$ Kai Chang, ${ }^{1,2}$ and Shu-Shen Li ${ }^{1,2}$ \\ ${ }^{1}$ SKLSM, Institute of Semiconductors, Chinese Academy of Sciences, P.O. Box 912, Beijing 100083, China \\ ${ }^{2}$ Synergetic Innovation Center of Quantum Information and Quantum Physics, \\ University of Science and Technology of China, Hefei, Anhui 230026, China \\ ${ }^{3}$ Department of Physics and Hebei Advanced Thin Film Laboratory, Hebei Normal University, Hebei 050024, China \\ ${ }^{4}$ School of Sciences, Hebei University of Science and Technology, Shijiazhuang, Hebei 050018, China \\ ${ }^{5}$ International Center for Quantum Materials and School of Physics, Peking University, Beijing 100871, China
}

(Dated: June 15, 2021)

\begin{abstract}
We investigate the scattering and localization properties of edge and bulk states in a disordered twodimensional topological insulator when they coexist at the same fermi energy. Due to edge-bulk backscattering (which is not prohibited a priori by topology or symmetry), Anderson disorder makes the edge and bulk states localized indistinguishably. Two methods are proposed to effectively decouple them and to restore robust transport. The first kind of decouple is from long range disorder, since edge and bulk states are well separated in $k$ space. The second one is from an edge gating, owing to the edge nature of edge states in real space. The latter can be used to electrically tune a system between an Anderson insulator and a topologically robust conductor, i.e., a realization of a topological transistor.
\end{abstract}

PACS numbers: 71.23.-k, 72.20.-i, 73.20.-r, 73.40.-c,

Introduction. What make topological insulators (TIs) unusual are the boundary states carrying dissipationless currents. This novel property is characterized by a topological invariant (e.g., Chern number or $Z_{2}$ invariant) associated with the occupied bulk bands. In the presence of boundaries (surfaces or edges), a nontrivial topological invariant guarantees the existence of gapless boundary states connecting the conduction and valance bands $\underline{1-3}$. In the bulk gap, the absence of backscattering between boundary states is protected by the topological order and some symmetry. Topological states in two-dimensional (2D) $)^{4-7}$ and three-dimensional (3D) $)^{8.9}$ systems have been experimentally observed recently.

However, the robust transport properties of boundary states are only valid without the interference of bulk states. Unfortunately, most of the 3DTIs found so far are actually metals, i.e., with fermi energy in the bulk band coexisting with boundary states 1.2 . This is one of the biggest experimental obstacles to realizing boundary transport in TIs. It has been observed that the mix between boundary and bulk states will lead to remarkable backscattering $1,9-11$. This backscattering can destroy the perfect conducting of boundary states. Therefore further understanding of boundary-bulk interplay is necessary. In Refs $\stackrel{12,13}{2}$, Fano-like rearrangements of bulk and boundary spectra arising from the mixing was discussed. Nevertheless, the scattering and transport properties due to disorder is not clear: how does such scattering happen? To what extent does it affect the robustness of boundary states? Most importantly, how can we avoid it?

In this paper, we systematically investigate the scattering between edge and bulk states in 2DTIs, in the presence of nonmagnetic impurities. We found that with the coexistence of edge and bulk states at the same fermi energy: 1) Anderson disorder tends to localize them indistinguishably; 2) Long range impurities can effectively decouple them and restore perfectly conducting channels (PCCs); 3) A local voltage gating at the edge is also enough to effectively decouple them and leads to PCCs, therefore gives rise to a convenient way to switch the system between a localized Anderson insulator and a perfect conducting TI. This is a concrete proposal of a topological field effect transistor (FET) ${ }^{14,15}$.

Model and methods. We adopt the Kane-Mele ${ }^{16}$ type model defined on a honeycomb lattice

$$
\begin{aligned}
H & =t \sum_{\langle i j\rangle, \sigma} c_{i \sigma}^{\dagger} c_{j \sigma}+\lambda_{v} \sum_{i, \sigma} \xi_{i} c_{i \sigma}^{\dagger} c_{i \sigma} \\
& +i \frac{\lambda_{\mathrm{SO}}}{3 \sqrt{3}} \sum_{\langle\langle i j\rangle\rangle, \sigma \sigma^{\prime}} v_{i j} c_{i \sigma}^{\dagger} s_{\sigma \sigma^{\prime}}^{z} c_{j \sigma^{\prime}},
\end{aligned}
$$

which has been used to describe the electronic states in silicene ${ }^{17-19}$. The first term describes the nearest-neighbor (NN) hopping, where $c_{i \sigma}^{\dagger}$ creates an electron at site $i$ with spin $\sigma$. The second term represents the staggered potential with $\xi_{i}= \pm 1$ for sublattice A (B). The third term is the intrinsic spin-orbital coupling (SOC) between the near-nearestneighbor (NNN) sites, where $s=\left(s_{x}, s_{y}, s_{z}\right)$ are the Pauli matrices for physical spins, and $v_{i j}=\left(\boldsymbol{d}_{i} \times \boldsymbol{d}_{j}\right)_{z} /\left|\boldsymbol{d}_{i} \times \boldsymbol{d}_{j}\right|= \pm 1$ with $\boldsymbol{d}_{i}$ and $\boldsymbol{d}_{j}$ the two NN bonds connecting NNN sites $i$ and $j$. Rashba SOC in realistic materials does not change the physics we will discuss, therefore it is not included for simplicity. Hereafter, we adopt $t$ as the energy unit and lattice constant $a$ (NNN distance) as the length unit.

The electronic structure of the model (1) has been well studied $^{18,19}$. The bulk bands are gapped at Dirac points $K\left(K^{\prime}\right)=( \pm 4 \pi / 3,0)$ with magnitude $\Delta_{G}=2\left|\lambda_{\text {SO }}-\lambda_{\mathrm{v}}\right|$. With finite gap $\Delta_{G}$, the system is a topologically trivial (nontrivial) insulator if $\lambda_{\text {SO }}<\lambda_{\mathrm{v}}\left(\lambda_{\mathrm{SO}}>\lambda_{\mathrm{v}}\right)$. In Fig. 1 (a), we plot the typical band structure of a quasi-one dimensional (1D) ribbon in the topological nontrivial phase. Within the bulk gap, $|E|<\left|\lambda_{\text {SO }}-\lambda_{\mathrm{v}}\right|$, there are four gapless edge states, corresponding to lower (L) and upper (U) edges, with spin up ( $\uparrow$ ) and down $(\downarrow)$, respectively. In the presence of time reversal symmetry (TRS) and sufficiently ribbon width, the backscat- 


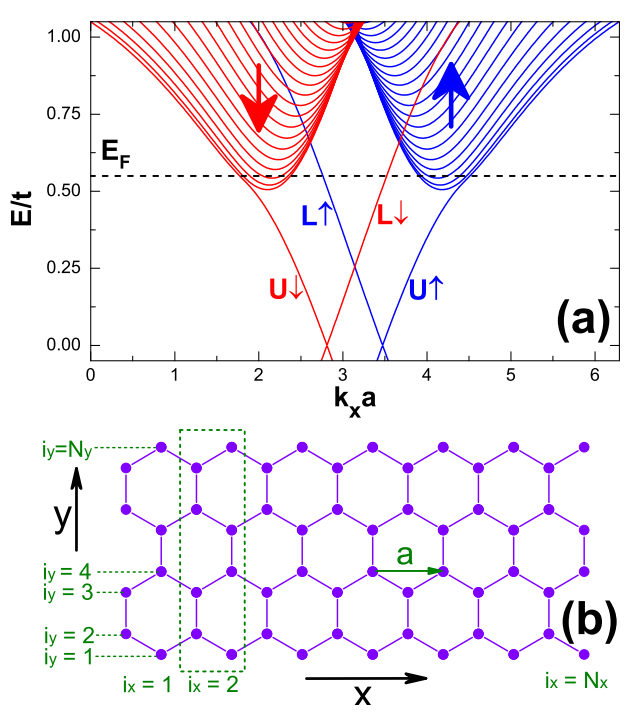

FIG. 1: (Color online) (a) Dispersion of a quasi-1D nanoribbon with zigzag edges and finite width $N_{y}=80$. The model parameters are: $\lambda_{\text {SO }}=0.8 t$ and $\lambda_{\mathrm{v}}=0.3 t$. States with spin $\uparrow(\downarrow)$ are plotted in blue (red). Edge states associated with lower (L) or upper (U) edge are also marked. (b) Schematic of the zigzag edge nanoribbon with length $N_{x}$ and width $N_{y}$. The unit cell is marked by the green dashed line.

tering between them is prohibited 20 . This is a typical model of quantum spin Hall effect.

The interests in the present work will be in the bulk bands. As shown in Fig. 1 (a), when $E>\lambda_{\text {SO }}-\lambda_{\mathrm{v}}=0.5 t$, one pair of edge states ( $\mathrm{U} \uparrow$ and $\mathrm{U} \downarrow$ ) at the upper edge merge into the bulk sates and lose their edge nature (hereafter they will be considered as bulk states), while another pair of edge states ( $\mathrm{L} \uparrow$ and $\mathrm{L} \downarrow$ ) at the lower edge survives. These have also been checked by calculating the wavefunction distributions in real space. In other words, model parameters are chosen such that there are edge states (well defined and distinguishable both in real space and in $k$ space) coexisting with the bulk bands at the same fermi energy. In the following, we will investigate the impurity scattering in this regime.

Non-magnetic impurities are expressed by adding a term to the Hamiltonian as

$$
H_{\mathrm{I}}=\sum_{i, \sigma} V_{i} c_{i \sigma}^{\dagger} c_{i \sigma},
$$

where $V_{i}$ is the random onsite potential, and is independent of spin to preserve TRS. Impurities induce scatterings between states. We investigate this in a standard geometry of quasi$1 \mathrm{D}$ ribbon infinitely extending in the $x$ direction, which is divided into three parts: the left lead (source), the central region (sample), and the right lead (drain). The leads are clean and semi-infinite, with well-defined channels indexed by $n$ at Fermi energy $E_{F}$. The width $N_{y}$ of the ribbon is chosen to be sufficiently large to avoid finite-size effects ${ }^{20}$. With impurities in the sample, the channel-resolved transmission amplitudes $T_{m n} \equiv T_{m \leftarrow n}$ between right-going channels, i.e., from channel $n$ in the left lead to channel $m$ in the right lead, can be calcu-
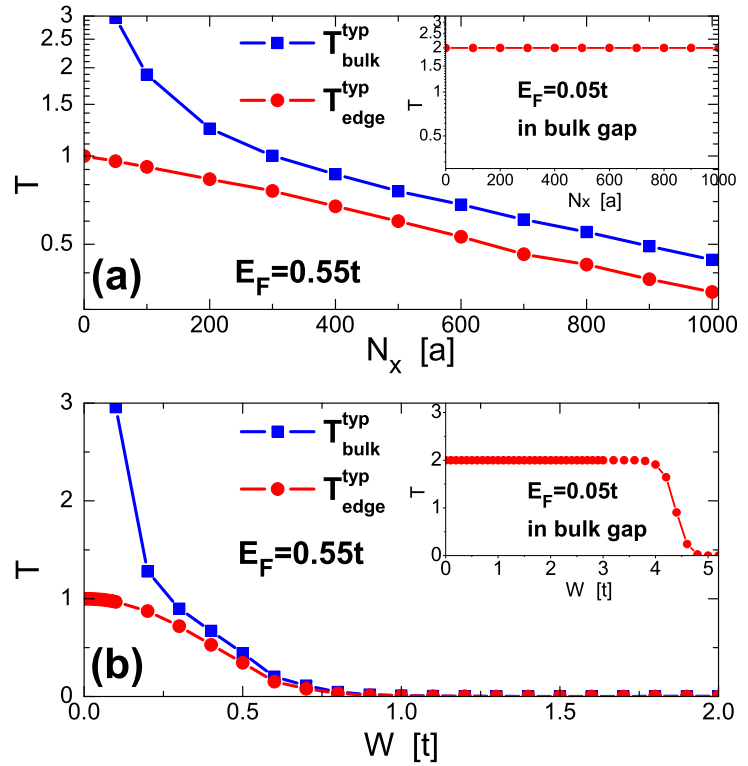

FIG. 2: (Color online) Typical values of bulk (blue square) and edge (red circle) transmissions for Anderson disorder, with ribbon width $N_{y}=80$. Main panels are for $E_{F}=0.55 t$ (in band) while insets are for $E_{F}=0.05 t$ (in bulk gap). (a) Transmissions as functions of sample length $N_{x}$ with $W=0.5 t$. (b) Transmissions as functions of disorder strength $W$ with $N_{x}=1000 a$. Other model parameters are the same as in Fig. 1 (a). Each data point is averaged over 1000 samples.

lated by using the numerical methods introduced in Refs ${ }^{21,22}$. The total transmission $\sum_{m n} T_{m n}$ is the conductance in units of $e^{2} / h$ at zero temperature, with $m$ and $n$ running over all rightgoing channels $\mathrm{s}^{23}$. The model parameters (except for disorder) are chosen to be identical for the sample and leads to reveal the intrinsic scattering behavior at this set of parameters.

In order to investigate the localization of edge states specifically, we define the edge transmission as

$$
T_{\text {edge }}=\sum_{m} \sum_{n \in \text { edge }} T_{m n}
$$

where $n$ only runs over edge channels in the left lead, and $m$ runs over all channels in the right lead. This $T_{\text {edge }}$ reflects the final fate of incident edge states after going through the sample. The bulk transmission $T_{\text {bulk }}$ can be defined similarly. Due to disorder, possible localization makes $T$ 's badly distributed over different disorder realizations. Thus it is essential to investigate the geometric mean $T^{\text {typ }}=\exp \langle\ln T\rangle$ to give the "typical value" instead of the arithmetic mean $T^{\text {ave }}=\langle T\rangle . \underline{24}$

Anderson impurities. In the presence of bulk-edge coexistence, a natural conjecture is that disorder will localize the bulk states and remain the edge states left. We will show that the case is not so simple. It has also been found that disorder can develop a topological phase from some definite Fermi energies in the bulk band ${ }^{25-27}$. Starting from a topologically nontrivial phase at clean limit, this just originates from a particlehole asymmetric band renormalization from disorder ${ }^{3,31}$. Our context is distinct from those that at clean limit, there exist 

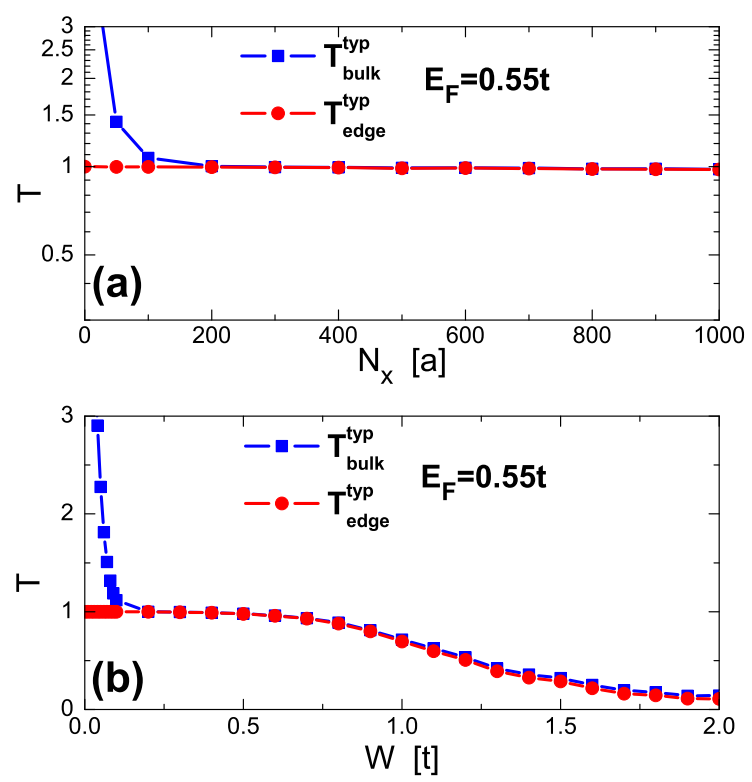

FIG. 3: (Color online) Same with main panels $\left(E_{F}=0.55 t\right)$ in Fig. 2 but for long range impurities $\xi=1.5 a, n_{I}=2.5 \%$. (a) Transmissions as functions of sample length $N_{x}$ with $W=0.5 t$. (b) Transmissions as functions of disorder strength $W$ with $N_{x}=1000 a$.

well-defined edge and bulk states at the Fermi energy, as well as particle-hole symmetry.

Let's start from Anderson disorder, where the random onsite potential $V_{i}$ are independently and uniformly distributed between $(-W / 2, W / 2)$. In the main panel of Fig. 2 (a), we show the bulk and edge transmissions as functions of sample length $N_{x}$, at fermi energy $E_{F}=0.55 t$ in the conduction band, a little above the band edge $E_{F}=0.5 t$. Compared to the robust in-gap edge transmission shown in the inset, the in-band transmissions are not robust and decay exponentially with increasing length. With the $T$-axis (transmission) in a logarithmic scale in Fig. 2] (a), the slope of the curve gives the inverse of localization length $\lambda$. It is now clear that edge states and bulk states localize with the same rate.

This non-robustness can also be seen from their dependence on disorder strength $W$, as shown in Fig. 2 (b): the in-band edge and bulk transmissions $\left(E_{F}=0.55 t\right.$, main panel) collapse to zero almost at the same time at $W \sim 0.8 t$, which are much earlier than the robust in-gap $\left(E_{F}=0.05 t\right.$, inset) edge transmission at $W \sim 5 t$. In this model, although the backscattering from the edge state $\mathrm{L} \downarrow$ (right-going) to the edge state $\mathrm{L} \uparrow$ (left-going) is still prohibited by TRS, the edge state L $\downarrow$ still decays by leaking into the bulk and being reflected to left going bulk channels. In other words, although there exist topological edge states, their leakage and backscattering into the bulk make the system into Anderson insulator, as normal disordered systems in 2D.

Long range impurities. The above results show the disastrous consequence of edge-bulk scattering in disordered 2DTI by destroying the robustness of edge states seriously. We are now in a position to look for means to avoid this. As can be seen in Fig. 10(a), the bulk and edge states are well separated in $k$ space when they coexist. To effectively decouple them, it is useful to consider the long range disorder, which suppresses scattering processes with large momentum transfer. In the presence of long range disorder, the onsite potential $V_{i}$ is the sum of contributions from $N_{I}$ impurities randomly centered at $\left\{\mathbf{r}_{m}\right\}$ among $N$ sites $V_{i}=\sum_{m=1}^{N_{I}} U_{m} \exp \left(-\left|\mathbf{r}_{i}-\mathbf{r}_{m}\right|^{2} /(2 \xi)\right)$, where $U_{m}$ is randomly distributed within $(-W / 2, W / 2)$. The above Anderson disorder corresponds to short range impurities with correlation length $\xi=+0$ and density $n_{i} \equiv N_{I} / N=100 \%$. Impurities with correlation length $\xi>a$ can be said to be long range, as widely investigated in graphene with substrate $\underline{28}-30$.

The transmissions for long range impurities $(\xi=1.5 a)$ are plotted in Fig. 3. In Fig. 3 (a), the edge transmission (red circle) almost does not decay with length at all, restoring its robustness by effective decoupling from bulk states. This robustness of the edge channel can also be seen from its dependence on disorder strength $W$ in Fig. 3 (b). On the other hand, the bulk transmission (blue square) decays very fast before $N_{x}<200$, reflecting the Anderson localization nature of the $2 \mathrm{D}$ bulk states. But after that this bulk decay also ceases at another robust channel $e^{2} / h$. This residual PCC can be attributed to the imbalance between the number of left- and right-moving bulk channels ${ }^{32}$, which is explained as follows.

The information of all active channels at $E_{F}=0.55 t$ are listed in Table [ The long range nature of disorder effectively decouples the edge channels (No. 8 for spin $\uparrow$ and No. 9 for spin $\downarrow$ ) from the bulk ones, which results in an imbalance between opposite channels in the bulk: there is one more right (left) going bulk channel for spin up (down) component, and there is no scattering between states with opposite spins due to TRS. Thus for a right going setup here, the residual right going spin-up channel exhibits itself by the appearance of a PCC. Notice this PCC in the bulk is a collective effect, and it does not corresponds to any one specific eigen-channel listed in table [1. Similar PCCs in other quasi-1D systems with odd number of channels have been discussed in Refs ${ }^{30,33}$.

\begin{tabular}{|c|c|c|c|c|c|c|c|c|}
\hline No. & 12 & 3 & 4 & 5 & 6 & 7 & & 8 \\
\hline velocity & - & - & - & + & + & + & & - \\
\hline spin & $\downarrow$ & $\downarrow$ & $\downarrow$ & $\downarrow$ & $\downarrow$ & $\downarrow$ & & $\begin{array}{c}\uparrow \\
\text { dge }\end{array}$ \\
\hline No. & 10 & 11 & 12 & 13 & 14 & 1. & & 16 \\
\hline velocity & + & - & - & + & + & + & & + \\
\hline spin & $\begin{array}{c}\downarrow \\
\text { edge }\end{array}$ & $\uparrow$ & $\uparrow$ & $\uparrow$ & $\uparrow$ & $\uparrow$ & & $\uparrow$ \\
\hline
\end{tabular}

TABLE I: Information for 16 conducting channels at $E_{F}=0.55 t$ as marked in Fig. 1 (a): the directions of their group velocities ( \pm for right (left) going) and the orientations of their spins. The numbers are sorted by ascending $k_{x}$.

Edge gating. The decoupling of edge and bulk states effectively recovers the robust conducting behavior of topological states. The above decoupling from long range disorder is based on the $k$ space consideration. However, the correlation length $\xi$ of impurities depends strongly on the details of materials, and is hard to control. Now we consider a real space proposal. For a 2DTI at the intrinsic Fermi energy $E_{F}=0.55 t$ 
in Fig. 1(a), a simple way to discard the redundant bulk states is to gate the whole 2D sample into the bulk gap, by a top gate covering the whole sample sheet. However, we will show that, an edge gating is also sufficient to create a decoupling between edge and bulk states and therefore result in PCCs. This is intuitive for a generalization to 3DTIs, because a gating to a 3D sample can only affect local carrier densities (therefore local chemical potentials) near the interface to the gate, instead of those in the whole bulk.

As illustrated in Fig. 4 (b), consider that a metal gate/insulator structure is attached to the lower edge $(y=0)$ of the 2DTI. This edge gate voltage $V_{E G}$ can only change the sample's local chemical potential $\mu$ around this edge, say, in an exponential decaying form as

$$
\mu(x, y)=E_{F}^{0}-\left(E_{F}^{0}-E_{G}\right) \exp \left(-y / \xi_{G}\right),
$$

where $E_{F}^{0}=\mu\left(y \gg \xi_{G}\right)$ is the intrinsic chemical potential (Fermi energy) in the bulk, $E_{G}=\mu(y=0)$ is the chemical potential at the gated edge $(y=0)$ determined by the edge gate $V_{E G}$, and $\xi_{G}$ is the decay length. If $E_{G}$ can be tuned into the bulk gap, and $\xi_{G}$ is larger than the penetration depth of the edge state in transverse direction, then there will be only edge state [the yellow arrow in Fig. 4(b)] in this gated region $\left(0 \leqslant y \lesssim \xi_{G}\right)$ near this edge, in spite of the presence of bulk states outside it. Although at different chemical potentials, the gated and ungated regions still belong to the same topological phase, with no additional edge states at the interface $\left(y \sim \xi_{G}\right)$ between them. So far, edge and bulk states have been well separated in real space. This decoupling should also restore the robustness of transport even for Anderson disorder. This is confirmed from the transmissions shown in Fig. 4(c), where perfectly conducting edge and bulk channels can be seen. The transmissions converge to $e^{2} / h$ rapidly when $E_{G}$ goes into the bulk gap and when $\xi_{G}$ is just several lattice constants long. We also show the transmissions at fixed length $N_{x}=1000 a$ when varying disorder strength $W$. As other proposals of edge tuning in $2 \mathrm{DTI}^{34-37}$, we have made this decoupling by taking advantage of the local nature of edge states.

Therefore, an edge gate can drive a disordered TI with bulkedge coexistence from Anderson insulator [Fig. 2] (a)] to a perfectly conductor [Fig. 4](c)]. This transition can be experimentally tuned to make a FET ${ }^{14,15}$. The onoff conductance ratio $\left.G_{\text {on }} / G_{\text {off }} \sim \exp \left[-N_{x} / \lambda_{G}\right] / \exp \left[-N_{x} / \lambda_{0}\right]\right]$, where $\lambda_{0}$ and $\lambda_{G}$ are the localization length of zero gating and edge gating respectively, as long as $N_{x}$ is within the phase coherence length. Moreover, the on state is very stable due to its topological origin. Since this tuning only requires an electric control near the boundary, it can be naturally generalized to 3D. Recently, a 3DTI based FET with boundary gating has been experimentally observed in ${ }^{38}$, and our present work offers a possible theoretical explanation.

Conclusions and discussions. All the above results are based on the Hamiltonian (1) for a topologically nontrivial phase $Z_{2}=1$. However, since this Hamiltonian does not contain Pauli matrices $s_{x}$ or $s_{y}$, it can be decoupled into spin $\uparrow$ and spin $\downarrow$ sub-systems, each of which is a Chern insulator with Chern number $\pm 1^{2,19}$. On the other hand, we have checked (but not shown here) that our results are also valid in the pres-
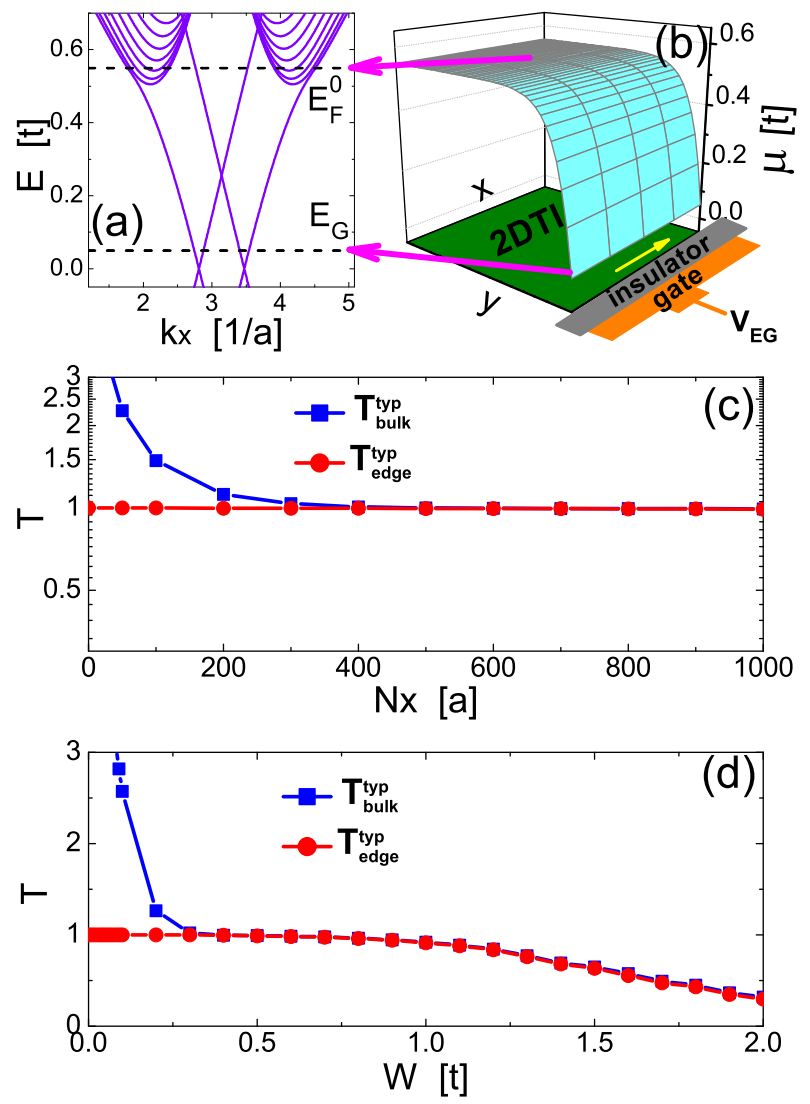

FIG. 4: (Color online) Edge gating. (a) Dispersion of the ribbon. (b) Illustration of the metal edge gate (orange)/insulator (gray) structure attached to one edge of the 2DTI (green), and the configuration of chemical potential $\mu(x, y)$ it induces. The electronic transport is along $x$-direction. The yellow arrow represents the edge state. (c) and (d) are all the same with main panels of Fig. 2 (a) and (b), but with an edge gating $E_{G}=0.05 t$ and $\xi_{G}=3 a$.

ence of Rashba SOC ${ }^{18,19}$, which couples spin $\uparrow$ and spin $\downarrow$. In one word, our conclusions can be generalized to Chern insulators and general $Z_{2} 2$ DTIs.

As a summary, we studied the edge and bulk transmissions of 2DTI when they coexist at the same fermi energy. Anderson disorder tends to localize edge and bulk states with the same localization length, because of unprotected backscattering between them. In order to decouple them and restore the robust transport of edge states, we introduced long range disorder and edge gating, based on momentum space and real space considerations, respectively. By edge gating, one can electrically tune the disordered system between an Anderson localized state and a robust extended state, making it a topological FET.

This work was supported by NSFC (grant nos. 11374294, 11204294, 11274364) and 973 Program Project No. 2013 CB933304. 
${ }^{1}$ M. Z. Hasan and C. L. Kane, Rev. Mod. Phys. 82, 3045 (2010).

2 X.-L. Qi and S.-C. Zhang, Rev. Mod. Phys. 83, 1057 (2011).

3 S.-Q. Shen, Topological Insulators: Dirac Equation in Condensed Matters ,(Springer Berlin 2012).

${ }^{4}$ M. König, S. Wiedmann, C. Brüne, A. Roth, H. Buhmann, L. W. Molenkamp, X. L. Qi and S. C. Zhang, Science 318, 766 (2007).

5 A. Roth, C. Brüne, H. Buhmann, L. W. Molenkamp, J. Maciejko, X.-L. Qi, S.-C. Zhang, Science 325, 294 (2009).

6 C.-Z. Chang, J.-S. Zhang, X. Feng, J. Shen, Z.-C. Zhang, M.-H. Guo, K. Li, Y.-B. Ou, P. Wei, L.-L. Wang, Z.-Q. Ji, Y. Feng, S.-H. Ji, X. Chen, J.-F. Jia, X. Dai, Z. Fang, S.-C. Zhang, K. He, Y.-Y. Wang, L. Lu, X.-C. Ma, Q.-K. Xue, Science 340, 167 (2013).

7 L.-J. Du, I. Knez, G. Sullivan, R.-R. Du, arXiv:1306.1925

8 Y. Xia, D. Qian, D. Hsieh, L. Wray, A. Pal, H. Lin, A. Bansil, D. Grauer, Y. S. Hor, R. J. Cava, and M. Z. Hasan, Nat. Phys. 5, 398 (2009).

9 D. Hsieh, D. Qian, L. Wray, Y. Xia, Y. S. Hor, R. J. Cava, and M. Z. Hasan, Nature 452, 970 (2008).

10 Y.-Y. Li, G. Wang, X.-G. Zhu, M.-H. Liu, C. Ye, X. Chen, Y.Y. Wang, K. He, L.-L. Wang, X.-C. Ma , H.-J. Zhang, X. Dai, Z. Fang, X.-C. Xie, Y. Liu, X.-L. Qi, J.-F. Jia, S.-C. Zhang, and Q.-K. Xue, Adv. Mater. 22, 4002 (2010).

11 S. Kim, M. Ye, K. Kuroda, Y. Yamada, E. E. Krasovskii, E. V. Chulkov, K. Miyamoto, M. Nakatake, T. Okuda, Y. Ueda, K. Shimada, H. Namatame, M. Taniguchi, and A. Kimura, Phys. Rev. Lett. 107, 056803 (2011).

12 D. L. Bergman and G. Refael, Phys. Rev. B 82, 195417 (2010).

13 Y.-T. Hsu, M. Fischer, T. L. Hughes, K. Park, and E.-A. Kim, arXiv:1401.3378.

14 Q.-K. Xue, Nat. Nano. 6, 197 (2011).

15 L. Andrew Wray, Nat. Phys. 8, 705 (2012).

16 C. L. Kane and E. J. Mele, Phys. Rev. Lett. 95, 146802 (2005).

17 C.-C. Liu, H. Jiang and Y. Yao, Phys. Rev. B 84, 195430 (2011).

${ }_{18}$ M. Ezawa, New J. Phys. 14, 033003 (2012).

19 M. Ezawa, Phys. Rev. Lett. 109, 055502 (2012).

${ }^{20}$ B. Zhou, H. Z. Lu, R. L. Chu, S. Q. Shen, and Q. Niu, Phys. Rev.
Lett. 101246807 (2008).

21 T. Ando, Phys. Rev. B 44, 8017 (1991).

22 P. A. Khomyakov, G. Brocks, V. Karpan, M. Zwierzycki, and P. J. Kelly, Phys. Rev. B 72, 035450 (2005).

23 Y. Imry and R. Landauer, Rev. Mod. Phys. 71, S306 (1999).

${ }^{24}$ K. Slevin, P. Markoš, and T. Ohtsuki, Phys. Rev. Lett. 86, 3594 (2001).

25 J. Li, R.-L. Chu, J. K. Jain and S.-Q. Shen, Phys. Rev. Lett. 102, 136806 (2009).

${ }^{26}$ H. Jiang, L. Wang, Q.-F. Sun, and X. C. Xie, Phys. Rev. B 80, 165316 (2009).

27 Y.-X. Xing, L. Zhang and J. Wang, Phys. Rev. B 84, 035110 (2011).

28 T. Ando and Nakanishi, J. Phys. Soc. Jpn. 67, 1704 (1998).

29 A. Rycerz, J. Tworzydło and C. W. J. Beenakker, Europhys. Lett. 79, 57003 (2007).

${ }^{30}$ K. Wakabayashi, Y. Takane and M. Sigrist, Phys. Rev. Lett. 99, 036601 (2007).

31 Y.-Y. Zhang and S.-Q. Shen, Phys. Rev. B 88, 195145 (2013).

32 S. D. Wang, Z.-Z. Sun, G. Xiong, S. Yin, and X R Wang, J. Phys. A: Math. Gen. 371337 (2004)

33 Y. Takane, S. Iwasaki, Y. Yoshioka, M. Yamamoto, and K. Wakabayashi, J. Phys. Soc. Jpn. 78034717 (2009).

34 Y.-T. Zhang, Q.-F. Sun and X. C. Xie, J. Appl. Phys. 109, 123718 (2011).

35 X.-T. An, Y.-Y. Zhang, J.-J. Liu, and S.-S. Li, New J. Phys. 14 083039 (2012).

36 H.-C. Li, L. Sheng and D. Y. Xing, Phys. Rev. Lett. 108196806 (2012).

37 H.-C. Li, L. Sheng, R. Shen, L. B. Shao, B.-G. Wang, D. N. Sheng, and D.Y. Xing, Phys. Rev. Lett. 110266802 (2013).

${ }^{38}$ H. Zhu, C. A. Richter, E. Zhao, J. E. Bonevich, W. A. Kimes, H.J. Jang, H. Yuan, H. Li, A. Arab, O. Kirillov, J. E. Maslar, D. E. Ioannou, and Q. Li, Sci. Rep. 3, 1757 (2013). 